\title{
A double suicide gene system driven by vascular endothelial growth factor promoter selectively kills human hepatocellular carcinoma cells
}

\author{
KAI WU, LIUCHENG YANG, ZONGHAI HUANG, HAIJUN ZHAO, JIANJUN WANG and SHUAI XU
}

Department of Surgery, Zhujiang Hospital, Southern Medical University, Guangzhou, Guangdong 510282, P.R. China

Received January 19, 2015; Accepted February 16, 2016

DOI: $10.3892 / 01.2016 .4360$

\begin{abstract}
The aim of the present study was to investigate the selective killing effect on hepatocellular carcinoma (HCC) cells of an adenovirus (Ad)-mediated cytosine deaminase (CD) in combination with thymidine kinase (TK) suicide gene system, driven by the vascular endothelial growth factor promoter (VEGFp), in vitro and in vivo. A double suicide gene system with VEGFp, named Ad-VEGFp-CDglyTK, was constructed and transfected into human HCC cells (BEL-7402 or HepG2; the latter cell type is deficient in VEGF) and human umbilical vein vascular endothelial cells (HUVEC). Green fluorescent protein expression was detected by fluoroscopy to verify transfection efficiency, and $C D g l y T K$ gene expression was detected by reverse transcription-polymerase chain reaction (PCR). The selective killing effect of Ad-VEGFp-CDglyTK was measured by 3-(4,5-dimethylthiazol-2-yl)-2,5-diphenyltetrazolium bromide assay and flow cytometry (FCM) in vitro and by xenograft studies in vivo. PCR revealed that the transgenic CDglyTK gene was expressed in BEL-7402 cells and HUVEC, but not in HepG2 cells. The cell survival rate significantly decreased in line with increasing concentrations of the prodrugs, ganciclovir (GCV) alone, 5-fluorocytosine (5-FC) alone or a combination of the two, in HUVEC and BEL-7402 cells with the transfected CDglyTK gene, but not in untransfected HUVEC or BEL-7402 cells, or in transfected or untransfected HepG2 cells. This result was additionally confirmed by FCM. GCV and 5-FC inhibited the HUVEC and BEL-7402 cells containing the transfected CDglyTK gene and also inhibited adjacent unmodified cells via the 'bystander effect'. No similar results were observed
\end{abstract}

Correspondence to: Dr Liucheng Yang, Department of Surgery, Zhujiang Hospital, Southern Medical University, 253 Middle of Gongye Avenue, Guangzhou, Guangdong 510282, P.R. China E-mail: zjhzh_1954@126.com

Key words: hepatocellular carcinoma, vascular endothelial growth factor, suicide gene, cytosine deaminase/ganciclovir, thymidine kinase/5-fluorocytosine in HepG2 cells. Compared with the control group, tumors with the transfected $C D g l y T K$ gene were smaller and the microvessel density of the tumor tissue was significantly decreased. It was concluded that a combination TK/GCV and $\mathrm{CD} / 5$-FC suicide gene system driven by VEGFp may provide a promising treatment strategy for HCC.

\section{Introduction}

Hepatocellular carcinoma (HCC) is the fifth most commonly observed malignant tumor globally, and the third leading cause of cancer-associated mortality (1-3). Surgery, chemotherapy, radiotherapy, immunotherapy and liver transplantation are typically used to treat patients with HCC (4). However, the 5-year age-standardized relative survival rates for $\mathrm{HCC}$ have not improved significantly over the past 10 years (5). Knowledge of the molecular mechanisms of HCC brings opportunities for therapeutic interventions against this type of cancer using novel approaches.

Suicide gene therapy, based on gene-directed enzyme prodrug therapy, is a promising alternative treatment to conventional chemotherapy. The suicide gene system makes use of an enzyme, which converts a non-toxic prodrug to its cytotoxic form (6). The combinations of the enzyme cytosine deaminase (CD) with the prodrug 5-fluorocytosine (5-FC) and of the enzyme thymidine kinase (TK) with the prodrug ganciclovir (GCV) are two of the most common suicide gene therapy systems $(7,8)$. In each case, the prodrug (5-FC or GCV) is non-toxic to normal human cells when administered on its own, but its enzyme (CD or TK, respectively) metabolizes the prodrug into a monophosphate form that subsequently acts as a chemotherapeutic agent against cancer cells (9). However, the current low efficiency of suicide gene systems limits their application (10). A number of studies have attempted to enhance the therapeutic effect of suicide gene therapy by combining it with other gene therapies $(11,12)$.

Vascular growth has a significant role in tumor development and metastasis (13). Anti-angiogenic therapy has been demonstrated to be safe and effective for the treatment of solid tumors (14). Vascular endothelial growth factor (VEGF), a critical proangiogenic regulator, is overexpressed in $\mathrm{HCC}$, whereas it has low levels of expression in normal liver tissues (15-17). Thus, VEGF is an ideal target for HCC treatment (18). 
In the present study, a combination suicide gene system using both CD and TK was utilized, along with a VEGF promoter (VEGFp), and this was delivered into HCC cells, followed by assessment of the in vitro and in vivo effects. The present study provides an experimental basis for additional application of double suicide gene therapy strategies.

\section{Materials and methods}

Cell lines. Human HCC cell lines (BEL-7402 and HepG2) and human embryonic kidney-293 (HEK-293) cells were obtained from the Animal Experimental Center of Sun Yet-Sen University (Guangzhou, China). Human umbilical vein vascular endothelial cells (HUVEC) were purchased from Nanjing KeyGen Biotech Co., Ltd. (Nanjing, China). Cells were cultivated in Dulbecco's modified Eagle's medium with $10 \%$ fetal bovine serum (both Gibco; Thermo Fisher Scientific, Inc. Waltham, MA, USA). All cells were maintained at $37^{\circ} \mathrm{C}$ in a humidified atmosphere containing $5 \% \mathrm{CO}_{2}$.

Construction of the recombinant plasmids. A $1.3 \mathrm{~kb}$ gene fragment encoding the $\mathrm{CD}$ gene and a $1.1 \mathrm{~kb}$ fragment encoding the TK gene were amplified by polymerase chain reaction (PCR) from Escherichia coli JM109 DNA and pREP8-TK, respectively (kindly provided by Cell biology department of Southern medical University, Guangzhou, China). These two DNA fragments were then inserted into the plasmid pMD18-T (catalog no., D101A; Takara Bio, Inc., Otsu, Japan) to generate pMD18-CD and pMD18-TK. Following confirmation by sequencing (Beijing Genomics Institution, Beijing, China), the CD and TK fragments were digested and inserted into pcDNA3 (provided by Department of Cell Biology, Southern medical University) to build the plasmid pcDNA3-CDglyTK. pcDNA3-CDglyTK was then cut by the restriction enzymes HindIII and PvuII (New England BioLabs, Inc., Ipswich, MA, USA) to generate CDglyTK-pA. The human VEGFp region was cut from pEGFP-1-SV-VEGFp (kindly provided by Professor Jiro Kishimoto, Shiseido Research Center, Yokohama, Japan) and amplified by PCR using the 7500 Fast Real-Time PCR system (Applied Biosystems; Thermo Fisher Scientific, Inc.). The cycling conditions were as follows: $98^{\circ} \mathrm{C}$ for $5 \mathrm{~min}$, followed by 28 cycles at $94^{\circ} \mathrm{C}$ for $40 \mathrm{sec}, 50^{\circ} \mathrm{C}$ for $40 \mathrm{sec}, 72^{\circ} \mathrm{C}$ for $1 \mathrm{~min}$ and $72^{\circ} \mathrm{C}$ for $10 \mathrm{~min}$. Subsequently, VEGFp was ligated to pAdtrack (Shanghai GenePharma Co., Ltd., Shanghai, China) to construct pAdtrack-VEGFp. Finally, CDglyTK-pA was added to generate pAdtrack-VEGFp-CDglyTK (Ad-VEGFp-CDglyTK). PCR primers are listed in Table I.

Amplification and identification of recombinant adenovirus. The vector (Ad-VEGFp-CDglyTK) was transfected into HEK-293 cells and purified using cesium chloride gradient ultracentrifugation at $10,000 \mathrm{x}$ g. Viral production and amplification were monitored with the aid of green fluorescent protein (GFP), and the titer of the purified recombinant adenoviruses was as high as $2.0 \times 10^{12} \mathrm{pfu} / \mathrm{ml}$. The recombinant adenovirus was verified by PCR.

Transfection efficiency. To measure the efficiency of transfection with Ad-VEGFp-CDglyTK, HUVEC and the human
HCC cell lines BEL-7402 and HepG2 (the latter is deficient in VEGF) were used. Cells were plated into six-well culture plates (Guangzhou RiboBio Co., Ltd., Guangzhou, China) at a density of $2 \times 10^{5}$ cells/well, and transfected with Ad-VEGFp-CDglyTK using various multiplicities of infection (MOI; 0, 10, 20, 50, 100, and $200 \mathrm{pfu} / \mathrm{cell}$ ). Fluorescence microscopy (GFM600 microscope, Leica Microsystems GmbH, Wetzlar, Germany) was used to assess GFP expression following 24 and $48 \mathrm{~h}$ of incubation at $37^{\circ} \mathrm{C}$.

Reverse transcription-polymerase chain reaction (RT-PCR). Total RNA was extracted from cells using TRIzol ${ }^{\circledR}$ reagent according to the manufacturer's protocol (Invitrogen; Thermo Fisher Scientific, Inc.) and digested with RNase-free DNase (Promega Corporation, Madison, WI, USA) to clear residual genomic DNA. The reverse transcription reaction was performed using the RevertAid ${ }^{\mathrm{TM}}$ First-Strand cDNA Synthesis kit (Fermentas; Thermo Fisher Scientific, Inc.) in a final volume of $20 \mu \mathrm{l}$ containing $2 \mu \mathrm{g}$ total RNA, $1 \mu \mathrm{g}$ oligo (dT) ${ }_{18}$ primer, $10 \mathrm{mmol} / \mathrm{l}$ deoxynucleotidetriphosphate mixture, $4 \mu \mathrm{l} 5 \mathrm{X}$ reverse transcription buffer, 20 units RiboLock ${ }^{\mathrm{TM}}$ Ribonuclease inhibitor, diethylpyrocarbonate-treated water and 200 units RevertAid ${ }^{\mathrm{TM}} \mathrm{M}-\mathrm{Mulv}$ reverse transcriptase. Following incubation at $42^{\circ} \mathrm{C}$ for $60 \mathrm{~min}$, the reverse transcription reaction was terminated by heating at $70^{\circ} \mathrm{C}$ for $10 \mathrm{~min}$. The reaction contained $2 \mu \mathrm{l}$ cDNA template. The cDNA was amplified by PCR using the 7500 Fast Real-Time PCR system (Applied Biosystems; Thermo Fisher Scientific, Inc.). The $25 \mu \mathrm{l}$ reaction mixture contained 2.5 units of Taq polymerase (Promega Corporation), $1 \mu 1$ of cDNA template (Promega Corporation), $1.5 \mathrm{mmol} / 1 \mathrm{MgCl}_{2}$ (Promega Corporation) and $0.5 \mu \mathrm{mol} / \mathrm{l} \mathrm{CDglyTK}$ primers. The CDglyTK primers were synthesized by Beijing Genomics Institution (forward, 5'-GGGAAGCTTAGGCTAGCAATGTCGAATAACGCT-3' and reverse, 5'-GGGTCTAGATTAGTTAGCCTCCCCCAT CTC-3'; generating a DNA fragment of 2,400 bp). Expression was normalized to the glyceraldehyde-3-phosphate dehydrogenase gene (forward, 5'-CTCAGACACCATGGGGAAGGT GA-3' and reverse, 5'-ATGACTTGAGGCTGTTGTCATA-3'; generating a DNA fragment of $450 \mathrm{bp}$; Beijing Genomics Institute, Beijing, China). The reaction conditions were as follows: Preliminary denaturation at $94^{\circ} \mathrm{C}(5 \mathrm{~min})$, followed by 30 cycles of denaturation at $94^{\circ} \mathrm{C}(40 \mathrm{sec})$, annealing at $58^{\circ} \mathrm{C}$ (60 sec) and extension at $72^{\circ} \mathrm{C}(90 \mathrm{sec})$, with a final extension step at $72^{\circ} \mathrm{C}$ for $10 \mathrm{~min}$. The PCR products were run on a $1.5 \%$ agarose gel, and examined on a CX2000 UV illuminator (UVP Inc., Upland, CA, USA) and photographed using a Canon EOS 60D camera (Canon, Inc., Tokyo, Japan). This experiment was performed three times.

\section{In vitro study}

Cell proliferation assay. To investigate the biological effect induced by suicide gene systems, cytotoxicity (the effect on cell viability) was assessed using MTT [3-(4,5-dimethylthiazol-2-yl)-2,5-diphenyltetrazolium bromide; Sigma-Aldrich, St. Louis, MO, USA] assay. HUVEC, BEL-7402, and HepG2 cells were seeded into 96-well plates (Guangzhou RiboBio Co., Ltd.) at a density of $5 \times 10^{3}$ cells/well for $24 \mathrm{~h}$. Following incubation at $37^{\circ} \mathrm{C}$ for $24 \mathrm{~h}$, the culture medium was removed, and cells were infected with Ad-VEGFp-CDglyTK 
Table I. Polymerase chain reaction primer sequences.

\begin{tabular}{lcl}
\hline Gene & Size, bp & \multicolumn{1}{c}{ Primers } \\
\hline CD & 1,300 & $\begin{array}{l}\text { Forward 5'-GGGAAGCTTAGGCTAGCAATGTCGAATAACGCT-3' } \\
\text { Reverse 5'-GGGGGATCCCTCCACGTTTGAATCGATGGCTTC-3' }\end{array}$ \\
TK & 1,100 & $\begin{array}{l}\text { Forward5'-GGGGGATCCGGCGGGGGCGGTGGAGGAGGGGGTATGGCTTCGTAC-3' } \\
\text { Reverse 5'-GGGTCTAGATTAGTTAGCCTCCCCCATCTC-3' }\end{array}$ \\
VEGFp & 572 & $\begin{array}{l}\text { Forward 5'-TCACCGCCTCGGCTTGTCACATCT-3' } \\
\text { Reverse 5'-ATGAACTTTCTGCTGTCTTGGGTG-3' }\end{array}$ \\
\hline
\end{tabular}

$\mathrm{CD}$, cytosine deaminase; TK, thymidine kinase; VEGFp, vascular endothelial growth factor promoter.

(100 pfu/cell) and incubated for $24 \mathrm{~h}$ at $37^{\circ} \mathrm{C}$. Uninfected cells incubated at $37^{\circ} \mathrm{C}$ for the same duration served as a control. Following incubation, the medium was replaced with various concentrations of GCV $(0,5,10,50,100$, or $200 \mu \mathrm{g} / \mathrm{ml}$; Roche Diagnostics GmbH, Mannheim, Germany) or 5-FC (0, 20, $40,60,80$, or $100 \mu \mathrm{g} / \mathrm{ml}$; Sigma-Aldrich), or a combination of the two. The cells were subsequently cultured for $48 \mathrm{~h}$ and incubated with $10 \mu \mathrm{l} \mathrm{MTT} \mathrm{(10} \mathrm{mg/ml;} \mathrm{Sigma-Aldrich).} \mathrm{The}$ medium was removed and the remaining purple-blue sediment was dissolved in $150 \mu$ l dimethyl sulfoxide (Sigma-Aldrich) for $10 \mathrm{~min}$. The relative optical density (OD) of each well was determined at the test wavelength $(490 \mathrm{~nm})$ using a Bio-Rad 2550 EIA Reader (Bio-Rad Laboratories, Inc., Hercules, CA, USA). Viability of the cells was calculated using the following equation: Cell survival rate $(\%)=(\mathrm{OD}$ value of experimental group / OD value of control group) x $100 \%$.

Bystander effect assay. Cells infected with recombinant virus were mixed with uninfected cells in various ratios (5:95, 10:90, 20:80, 40:60, 60:40, and 80:20) and seeded into 96-well plates at $1 \times 10^{4}$ cells/well. Cells were treated with GCV $(100 \mathrm{mg} / \mathrm{l})$ and 5-FC $(80 \mathrm{mg} / \mathrm{l})$ together for $48 \mathrm{~h}$ and analyzed by MTT assay, as described above.

Flow cytometry analysis. To determine the effect of recombinant viruses on apoptosis, Annexin V-fluorescein isothiocyanate (FITC) and propidium iodide (PI; BD Biosciences, Franklin Lakes, NJ, USA) were used for flow cytometry (FCM) analysis to assess apoptosis. Cells infected with recombinant virus and uninfected cells were treated with GCV (100 mg/l), 5-FC ( $80 \mathrm{mg} / \mathrm{l})$ or a combination of the two for $24 \mathrm{~h}$. The harvested cells were washed twice with phosphate-buffered saline (PBS), and adjusted to a concentration of $5 \times 10^{5}$ cells $/ \mathrm{ml}$ by dilution with PBS. The cell suspension (200 $\mu \mathrm{l}$ ) was added to each labeled tube, followed by $5 \mu \mathrm{l}$ of Annexin V-FITC and $10 \mu \mathrm{l}$ PI. The tube was incubated for at least $10 \mathrm{~min}$ at room temperature in the dark. Cells were assayed using a FACSCalibur ${ }^{\mathrm{TM}}$ Flow Cytometer (BD Biosciences). Apoptotic cells were defined as the population that were Annexin V-FITC positive and PI-negative.

\section{In vivo study}

Tumor cell xenograft. Animal experiments were approved by the Institutional Animal Care and Use Committee of Southern Medical University (Guangzhou, China). In total, 20 male nude mice (4-6 weeks of age, 18-20 g) were purchased from the Laboratory Animal Center of Sun Yet-Sen University. The mice were kept in a specific-pathogen-free room at $26-28^{\circ} \mathrm{C}$ in a $10 \mathrm{~h}$ light and 14 dark cycle, and had free access to food and water. The mice were randomly divided into four groups with 5 mice in each group. The BEL-7402 cell suspension (either infected with Ad-VEGFp-CDglyTK or uninfected) was injected subcutaneously $\left(0.5 \times 10^{7}\right.$ cells in $200 \mu \mathrm{l}$ serum-containing medium). Mice were observed daily to ensure that the injection site was healthy. GCV (200 mg/kg/day) and 5-FC (100 mg/kg/day) were injected into the tumor daily for 10 days. At 1 week subsequent to injection, tissue samples and other biological data (tumor weight, and long and short diameter) were collected. The tumor volume was calculated using the following formula: Volume $=\left(\mathrm{axb}^{2}\right) / 2 \mathrm{~mm}^{3}$ (a, longer diameter of the tumor; b, shorter diameter of the tumor) (19). The rate of inhibition of tumor growth was subsequently calculated as the tumor inhibition rate as follows: (1-tumor weight of treatment group/tumor weight of control group)x $100 \%$.

Microvessel density assay. To assess tumor angiogenesis, cluster of differentiation 34 antibody was used to determine microvessel density (MVD). Tumor tissue was collected and fixed in $10 \%$ formaldehyde (Shanghai Chemical Reagent Co., Ltd., Shanghai, China) for $24 \mathrm{~h}$. The tumor tissue was embedded in paraffin and $4-\mu \mathrm{m}$ sections were cut using the RM2245 microtome (Leica Microsystems GmbH). To block endogenous peroxidase activity, $3 \% \mathrm{H}_{2} \mathrm{O}_{2}$ (Shanghai Chemical Reagent Co., Ltd.) was added for $10 \mathrm{~min}$. Following antigen retrieval with sodium citrate $(0.01 \mathrm{mmol} / 1, \mathrm{pH} 6.0$; Shanghai Chemical Reagent Co., Ltd.) for $10 \mathrm{~min}$, rabbit anti-human cluster of differentiation 34 monoclonal antibody (catalog no., sc-19621; 1:150 dilution; Santa Cruz Biotechnology, Inc., Dallas, TX, USA) was incubated with samples overnight at $4^{\circ} \mathrm{C}$, followed by incubation with the secondary biotin-labeled goat anti-mouse immunoglobulin $\mathrm{G}$ antibody (1:500 dilution; catalog no., A0286; Beyotime Institute of Biotechnology, Haimen, China) at $37^{\circ} \mathrm{C}$ for $30 \mathrm{~min}$. Following staining with 3,3'-diaminobenzidine (OriGene Technologies, Inc., Beijing, China) and hematoxylin (Shanghai Chemical Reagent Co.), MVD was assessed under a microscope (TS100; Nikon Corporation, Tokyo, Japan), as described previously by Weidner et al (20). Under x100 magnification, five areas were identified with the highest vascular density ('hot spots'), and the number of vessels in each of these regions was counted 

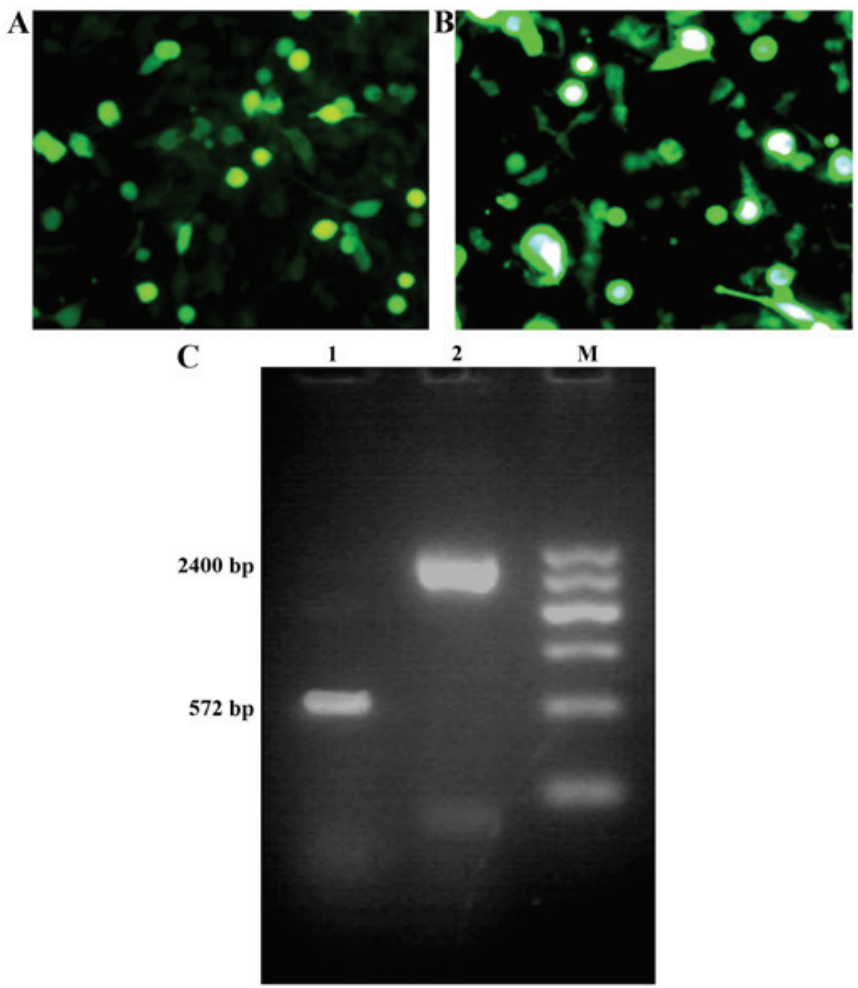

Figure 1. Transient expression of the recombinant GFP genes was observed by fluorescence microscopy (A) $24 \mathrm{~h}$ and (B) $48 \mathrm{~h}$ post-transfection (MOI, $100 \mathrm{pfu} / \mathrm{cell}$; magnification, x200). (C) Reverse transcription-polymerase chain reaction was used to confirm the recombinant adenovirus in HEK-293 cells. Lane M, Marker; Lane 1, transfected Ad-VEGFp-CDglyTK HEK-293 cells revealing the presence of the 572 bp target band (VEGFp); Lane 2, transfected Ad-VEGFp-CDglyTK HEK-293 cells showing the presence of the 2400 bp target band (CDglyTK). VEGFp, vascular endothelial growth factor promotor; $\mathrm{CD}$, cytosine deaminase; TK, thymidine kinase; Ad, adenovirus.

under x200 magnification. Microvessels were counted by two independent observers, and the mean value was used for analysis.

Statistical analysis. Each assay was performed at least three times, and data are presented as the mean \pm standard deviation. Analysis of variance was used to determine the significance of differences in multiple comparisons. All data were analyzed with SPSS (version 13.0; SPSS Inc., Chicago, IL, USA). P<0.05 was considered to indicate a statistically significant difference.

\section{Results}

Recombinant viruses. At $24 \mathrm{~h}$ following transfection of recombinant virus plasmids into HEK-293 cells, a stronger GFP fluorescence signal was observed in the cells transfected with Ad-VEGFp-CDglyTK, while the majority of the remaining HEK-293 cells expressed GFP protein at $48 \mathrm{~h}$ post-transfection. The recombinant adenoviruses were confirmed by PCR and DNA electrophoresis, producing bands of 572 bp (same size as VEGF) and 2,400 bp (same size as the CDglyTK gene fragment; Fig. 1).

Transfection efficiency of Ad-VEGFp-CDglyTK in various cell lines. To determine the infection efficiency of the adenoviral
A

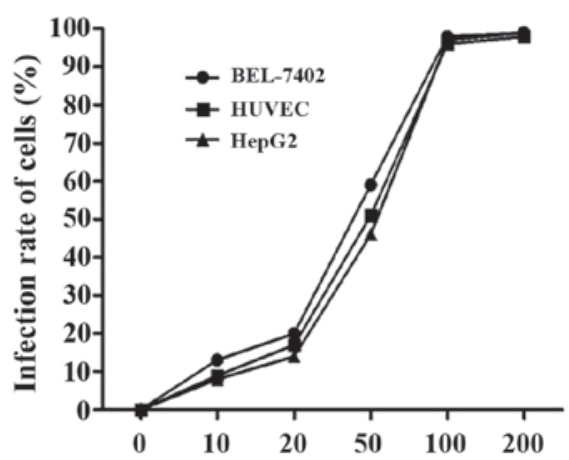

B

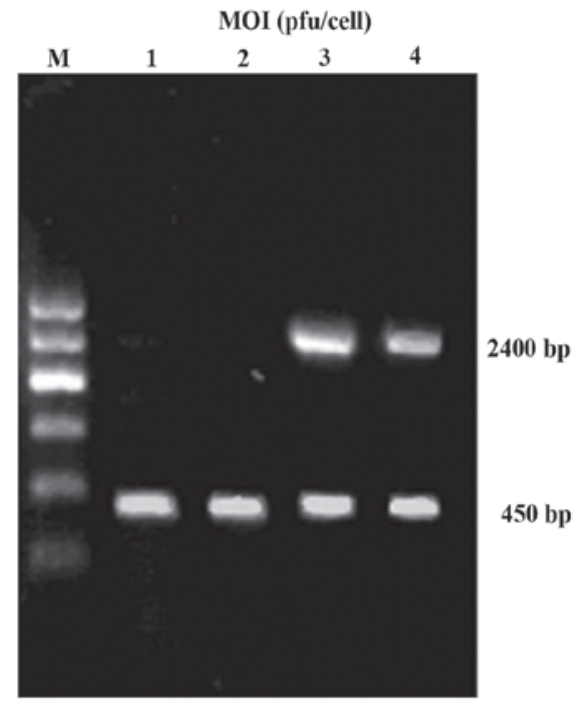

Figure 2. Recombinant adenovirus transfer efficiency and CDglyTK production in various cell lines. (A) Transfer efficiencies of recombinant adenoviruses in various cell lines. When the MOI was $100 \mathrm{pfu} / \mathrm{cell}$, the transfer efficiency was $95 \%$ in all three cell lines. (B) Reverse transcription-polymerase chain reaction analysis of the expression of CDglyTK lane (DNA fragment of $2400 \mathrm{bp}$ ). Glyceraldehyde-3-phosphate dehydrogenase was used as an internal control (DNA fragment of $450 \mathrm{bp}$ ). Lane M, Marker; Lane 1, blank control group; Lane 2, no fragments of CDglyTK gene detected in HepG2 cells; Lane 3, CDglyTK gene detected in BEL-7402 cells; Lane 4, CDglyTK gene detected in HUVEC. MOI, multiplicity of infection; CD, cytosine deaminase; TK, thymidine kinase; HUVEC, human umbilical vein vascular endothelial cells.

vector, the BEL-7402, HUVEC and HepG2 cells were infected with the Ad-VEGFp-CDglyTK at a MOI ranging from 10-200 pfu/cell. At a MOI of $10 \mathrm{pfu} /$ cell, only a small number of cells expressed GFP, whereas at a MOI of $100 \mathrm{pfu} / \mathrm{cell}$, $>95 \%$ of cells were GFP-positive without demonstrating any marked adenoviral toxicity (Fig. 2A). To investigate expression of the CDglyTK gene in the infected cells, RNAs were analyzed by RT-PCR. It was observed that the $C D g l y T K$ fusion gene was expressed in the BEL-7402 cells and HUVEC, but not in HepG2 cells (Fig. 2B).

Cytotoxicity analysis of the Ad-VEGFp-CDglyTK on transfected cells in vitro. To investigate the biological effect induced by Ad-VEGFp-CDglyTK, cytotoxicity was assessed in HUVEC, BEL-7402, and HepG2 cells infected with Ad-VEGFp-CDglyTK and treated with GCV, 5-FC or GCV+5-FC. As shown in Fig. 3A, HUVEC and BEL-7402 cells were highly sensitive to the prodrugs, but HepG2 cells were 
A

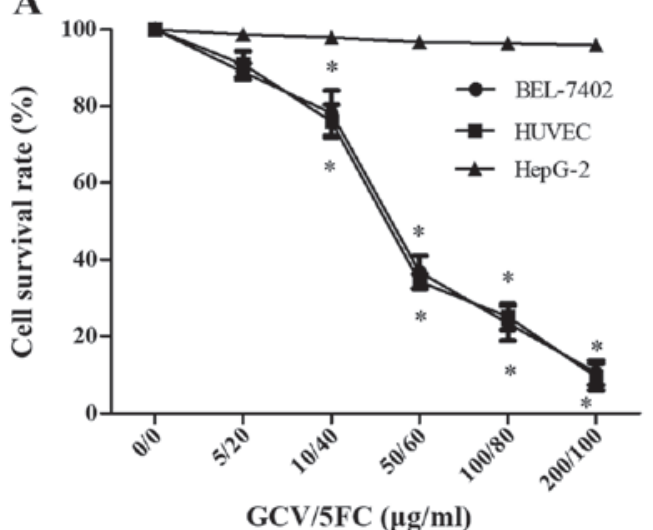

C

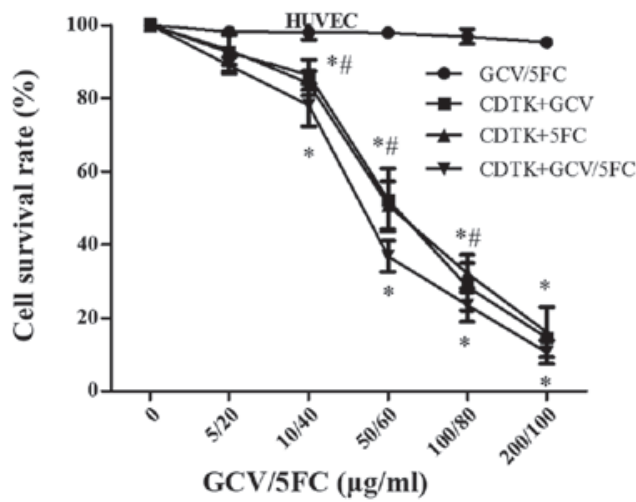

B

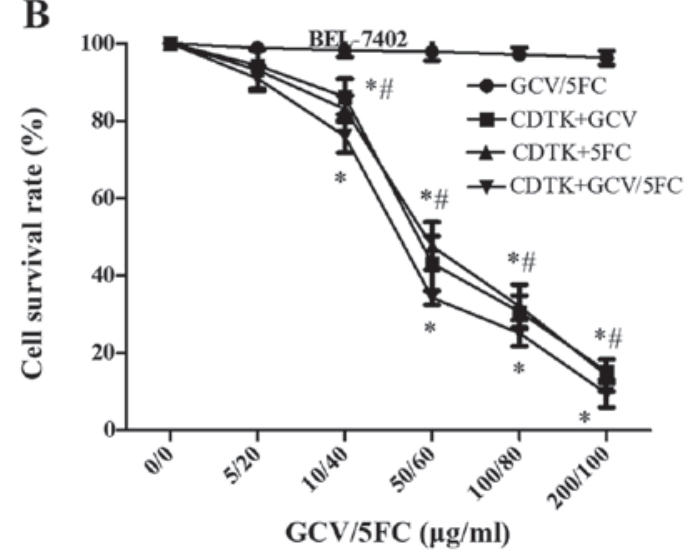

D

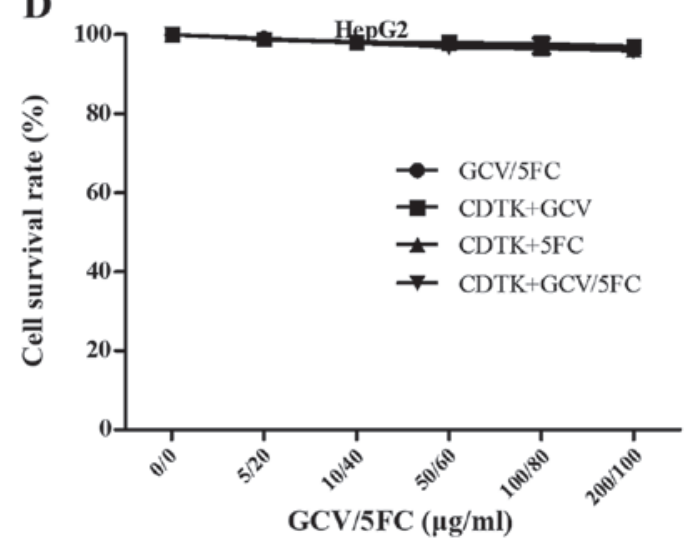

Figure 3. Effect of the recombinant adenoviruses on the cell survival rate of various transgenic cells. (A) Influence on survival rate of HUVEC, BEL-7402 and HepG2 cells of various concentrations of GCV combined with 5-FC. "P<0.05 compared with HepG2 cells. (B) Sensitivity of BEL-7402 cells and BEL-7402 cells transfected with $C D$ glyTK cells to various concentrations of GCV, $5-\mathrm{FC}$ or a combination of the two. ${ }^{*} \mathrm{P}<0.05$ compared with the untransfected group; ${ }^{*} \mathrm{P}<0.05$ GCV or 5-FC alone compared with GCV+5-FC. (C) Sensitivity of HUVEC and HUVEC transfected with CDglyTK cells to various concentrations of GCV, 5 -FC or a combination of the two. ${ }^{*} \mathrm{P}<0.05$ compared with the untransfected group; ${ }^{*} \mathrm{P}<0.05$ GCV or 5 -FC alone compared with GCV+5-FC. (D) Sensitivity of HepG2 cells and HepG2 cells transfected with CDglyTK cells to various concentrations of GCV, 5-FC or a combination of the two. HUVEC, human umbilical vein vascular endothelial data; GCV, ganciclovir; 5-FC, 5-fluorocytosine; CD, cytosine deaminase; TK, thymidine kinase.

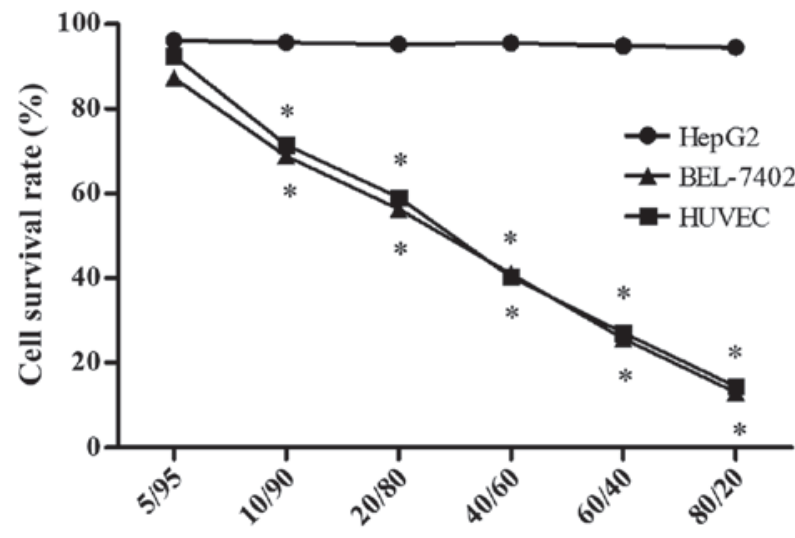

Transfected/untransfected CDTK cells ratio

Figure 4. Bystander effects of prodrugs (ganciclovir+5-fluorocytosine) on BEL-7402 cells, HUVEC and HepG2 cells with various proportions of transfected and untransfected cells. " $\mathrm{P}<0.05$ compared with HepG2 cells HUVEC, human umbilical vein vascular endothelial data; $C D$, cytosine deaminase; TK, thymidine kinase.

not sensitive to the prodrugs $(\mathrm{P}=0.0006)$. Cell survival rates significantly decreased in line with increasing concentrations of the prodrugs in the GCV+5-FC group and in the individual prodrug treatment groups $(\mathrm{P}=0.027)$. The sensitivity of CDglyTK-transfected cells to $\mathrm{GCV}+5-\mathrm{FC}$ was greater compared with their sensitivity to GCV or 5-FC alone in the BEL-7402 and HUVEC groups ( $\mathrm{P}=0.013$; Fig. 3B and $\mathrm{C}$ ); however, there was no significant difference in the sensitivity of HepG2 cells to either of the single drugs or to the combination treatment $(\mathrm{P}=0.923$; Fig. 3D).

Bystander effect of Ad-VEGFp-CDglyTK. The bystander effect of the $C D g l y T K$ gene was assessed by mixing transfected and untransfected cells in various ratios. In the BEL-7402 and HUVEC groups, the survival rate decreased significantly as the proportion of Ad-VEGFp-CDglyTK-transfected cells increased ( $\mathrm{P}=0.041$; Fig. 4); however, the HepG2 cells did not exhibit this phenomenon $(\mathrm{P}=0.718)$. In addition, cell survival rates were all markedly lower in the transfected cells compared with the untransfected cells for HUVEC and BEL-7402 cells, but not for HepG2 cells. The cell survival rate was $\sim 81 \%$ at a ratio of $95 \%$ untransfected to $5 \%$ transfected BEL-7402 cells, but when the proportion of untransfected cells was reduced to $90 \%$, the cell viability fell to $69 \%$. These results indicate that GCV and 5-FC are able to kill transfected cells, but are additionally able to kill untransfected cells via a bystander effect. 
A

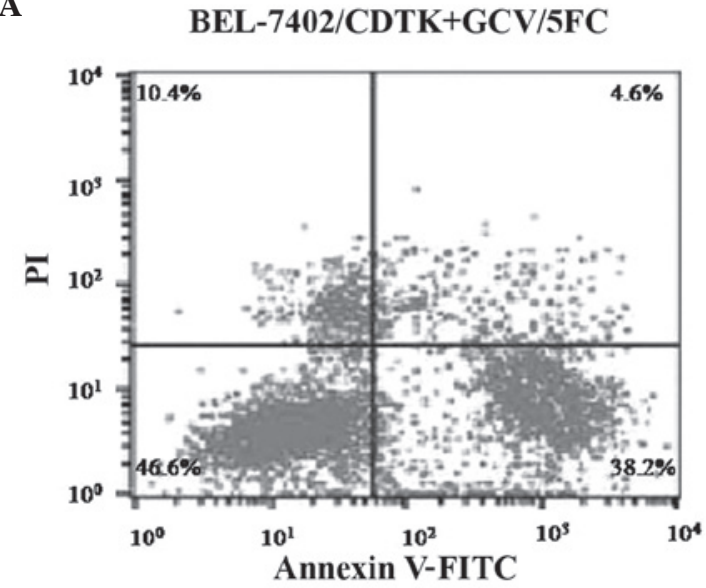

C

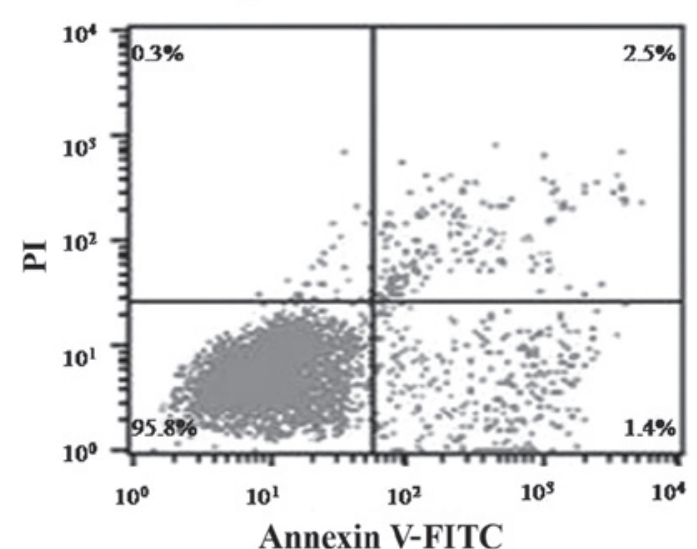

B

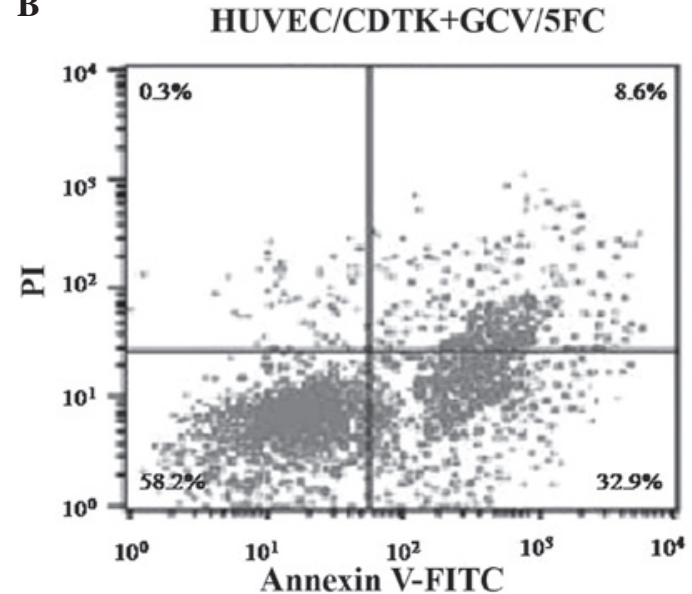

D

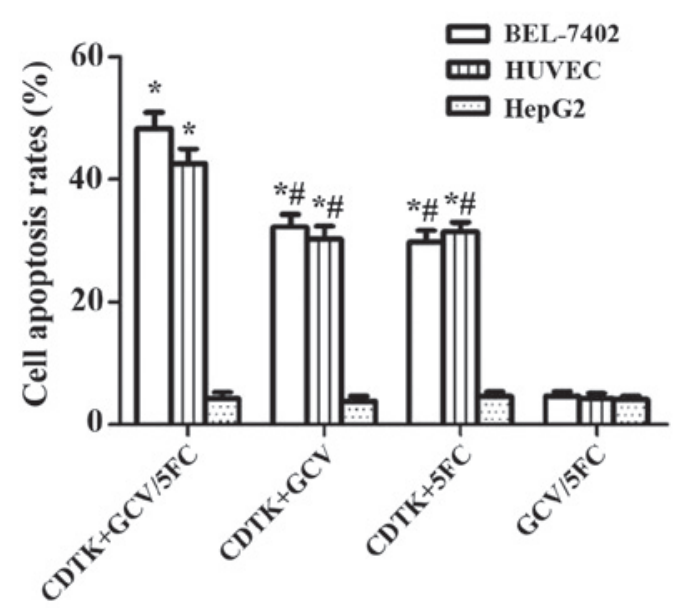

Figure 5. Flow cytometric analysis of the effect of GCV combined with 5-FC on cell apoptosis. Cell lines transfected with Ad-VEGFp-CDglyTK; (A) BEL-7402; (B) HUVEC and (C) HepG2 cells. (D) Cell apoptosis rate induced by the prodrugs GCV and/or 5-FC in the cell lines BEL-7402, HUVEC and HepG2. ${ }^{*} \mathrm{P}<0.05$ compared with the untransfected group; ${ }^{\#} \mathrm{P}<0.05 \mathrm{GCV}$ or 5-FC alone compared with GCV+5-FC. GCV, gancivlovir; 5-FC, 5-fluorocytosine; VEGFp, vascular endothelial growth factor promotor; CD, cytosine deaminase; TK, thymidine kinase; HUVEC, human umbilical vein vascular endothelial data; PI, propidium iodide; FITC, fluorescein isothiocyanate; Ad, adenovirus.

Flow cytometric analysis. To additionally analyze the effect of Ad-VEGFp-CDglyTK on tumor cells, cell apoptosis was assessed by FCM. As demonstrated in Fig. 5, apoptosis rates were significantly increased $(\mathrm{P}<0.0001)$ in the Ad-VEGFp-CDglyTK-transfected BEL-7402 cells and HUVEC compared with the HepG2 cells. The apoptosis rate was additionally higher in the recombinant virus-transfected group compared with the untransfected groups for the BEL-7402 cells and HUVEC ( $\mathrm{P}=0.012)$, but not for the HepG2 cells $(\mathrm{P}=0.872)$. The combination of GCV+5-FC exerted a stronger effect than either drug alone ( $\mathrm{P}=0.023$; Fig. 5D).

Anti-tumor effect of Ad-VEGFp-CDglyTK in vivo. Based on the results of cytotoxicity and FCM analysis, BEL-7402 cells, with or without the Ad-VEGFp-CDglyTK system, were injected subcutaneously into nude mice. As demonstrated in Fig. 6, the tumor cells transfected with recombinant virus formed smaller tumors compared with the untransfected cells, and the volumes of the tumors were significantly smaller for the transfected group compared with the untransfected group $(\mathrm{P}=0.0003)$. The inhibition rate was increased for the Ad-VEGFp-CDglyTK cells treated with GCV+5-FC group compared with the untransfected group $(\mathrm{P}=0.003)$. Therefore, combination of the recombinant virus with the prodrugs $(\mathrm{GCV}$, 5-FC or GCV+5-FC) was able to suppress the growth of HCC cells significantly in vivo. The GCV+5-FC combination exerted a stronger tumor-suppressor effect than either drug alone $(\mathrm{P}<0.001)$. It is notable that this difference was more marked in the in vivo experiment compared with the in vitro studies.

Effect of Ad-VEGFp-CDglyTK on MVD of HCC in nude mice. The MVD of the tumor tissue was assessed by cluster of differentiation 34 immunohistochemistry. The MVD of the tumor tissue was decreased more significantly $(\mathrm{P}=0.0008)$ in the group transfected with Ad-VEGFp-CDglyTK and treated with prodrugs (GCV, 5-FC, or GCV+5-FC) compared with the control group (Fig. 7). The MVD was slightly but significantly $(\mathrm{P}=0.012)$ lower in the GCV+5-FC group compared with the groups treated with either drug alone.

\section{Discussion}

Chemotherapy is an established and successful therapy for retarding the growth of a variety of tumor types (4). However, 
A

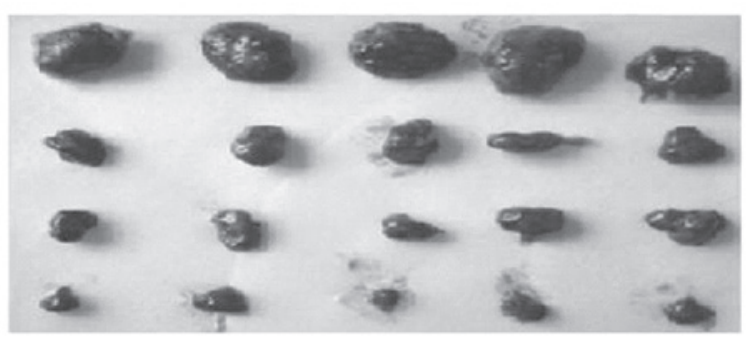

BEL-7402+GCV/5FC

BEL-7402/CDTK+GCV

BEL-7402/CDTK+5FC

BEL-7402/CDTK+GCV/5FC

B
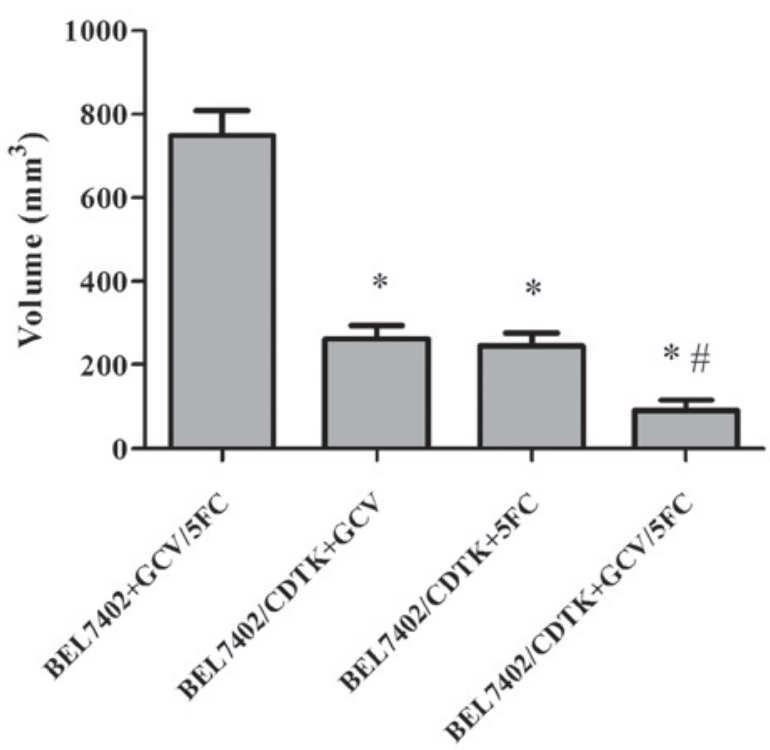

Figure 6. Tumor formation in BEL7402 cells was treated with 5-FC, GCV or a combination of the two for 14 days. Tumors in the suicide gene transfection group were significantly (A) smaller in size and (B) lower in volume compared with those in the untransfected group. "P $<0.05$ transfected group compared with the untransfected group; ${ }^{\mathrm{P}}<0.05 \mathrm{GCV}$ or 5-FC alone compared with GCV+5-FC. 5-FC, 5-fluorocytosine; GCV, gancivlovir; $\mathrm{CD}$, cytosine deaminase; TK, thymidine kinase.

the majority of chemotherapy drugs are not cancer-specific and may cause off-target organ toxicity (21). Suicide gene therapy, using enzymes that are not toxic to healthy tissues but that produce highly toxic metabolites from a much less toxic prodrug, is a safe and efficient therapeutic option for cancer (22). As reported in several studies, TK/GCV or $\mathrm{CD} / 5$-FC are the longest established of these suicide gene therapy systems (23-25). Single suicide gene systems (using one prodrug and one enzyme) are able to inhibit tumor development, but a number of studies have demonstrated that TK/GCV combined with CD/5-FC has a higher efficacy for the treatment of solid tumors compared with single suicide gene systems $(26,27)$. However, the suicide gene systems investigated in the present study (TK/GCV and CD/5-FC) have demonstrated little efficacy in clinical practice, due to their low targeting and poor gene-transfer efficiencies in tumors (28). Therefore, a gene that is able to improve the targeting and gene-transfer efficiencies of these systems is urgently required.

Angiogenesis, the formation of new blood vessels from existing vasculature, is an important process in numerous malignancies, including HCC (29). VEGF is a critical proangiogenic factor that has a significant role in the invasion and metastasis of HCC (30). A number of studies have verified that VEGF expression levels are increased in vascular endothelial cells of HCC compared with those of normal tissues $(31,32)$. A meta-analysis additionally revealed that the

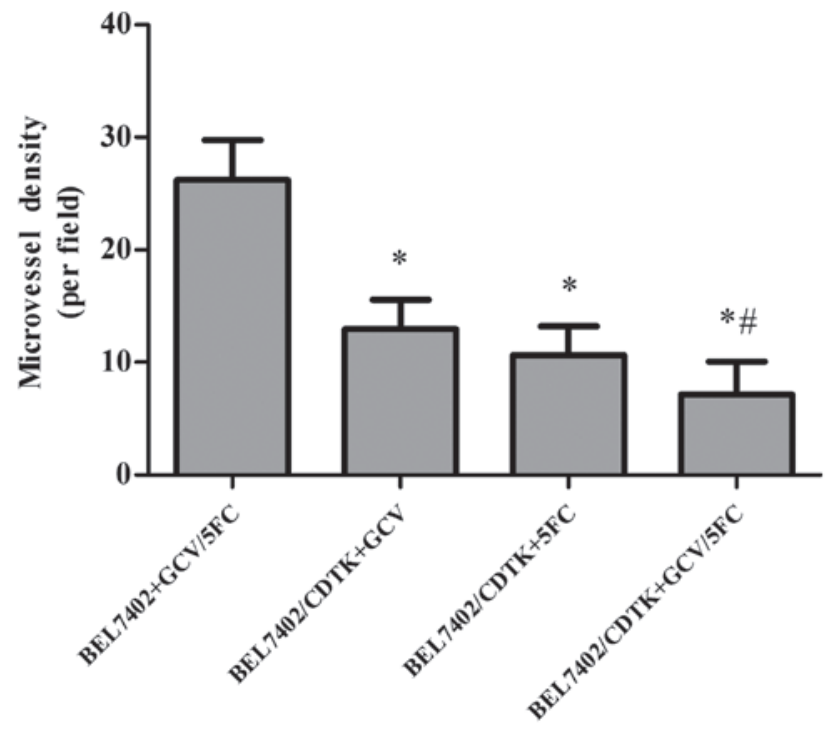

Figure 7. Effect of Ad-VEGFp-CDglyTK on the microvessel density of human hepatocellular carcinoma tumors in nude mice. ${ }^{*} \mathrm{P}<0.05$ transfected group compared with the untransfected group; ${ }^{~} \mathrm{P}<0.05 \mathrm{GCV}$ or 5 -FC alone compared with GCV+5-FC. VEGFp, vascular endothelial growth factor promotor; $\mathrm{CD}$, cytosine deaminsase; TK, thymidine kinase; GCV, gancivlovir; 5-FC, 5-fluorocytosine; Ad, adenovirus.

serum VEGF level is associated with prognosis for patients with HCC (33). Inhibiting angiogenesis by using anti-VEGF has been proposed as a potential anticancer strategy (34). 
Therefore, the VEGF promoter has been utilized to express target genes in HCC due to its tumor-specific expression (32). In the current study, the results demonstrated that VEGFp was able to direct the CDglyTK gene in VEGF-high expressing cells (BEL-7402 and HUVEC), but the transgenic CDglyTK double suicide genes were not expressed in HepG2 cells owing a deficiency in VEGF. Therefore, this suggests that when the VEGFp-driven suicide gene is systemically administered, the systemic toxicity to normal cells may be significantly reduced compared with that of traditional chemotherapy.

In the present cytotoxicity experiment, BEL-7402 cells and HUVEC with high levels of CDglyTK expression were highly sensitive to the prodrugs used (GCV, 5-FC and a combination of the two). These prodrugs significantly decreased the survival rate of BEL-7402 cells and HUVEC, and the cytotoxic effect increased in line with increasing drug dose. However, such inhibition was not observed in HepG2 cells, which have low CDglyTK expression. Previous studies indicated an increased killing efficiency of a combination suicide gene system compared with any single suicide gene system, due to the synergetic cytotoxicity of the combined gene system $(6,35)$. Furthermore, Su et al (8) indicated that the effect of double suicide genes was much stronger than that of individual suicide genes in breast cancer cells. Although the present study used different genes and cancer cells, the experiments of the current study also revealed that although each single prodrug (GCV or 5-FC) was able to kill tumor cells in the transgenic CDglyTK BEL-7402 cells and HUVEC, the combination of these two prodrugs exerted a more powerful killing effect $(\mathrm{P}<0.05)$. Thus, combination treatment may reduce the drug dose required and additionally decrease the toxic side effects of suicide gene systems on other organs.

The bystander effect is the primary driving force of the suicide gene therapy strategy $(36,37)$. The results of the present study demonstrated that GCV+5-FC was not only able to kill the transfected cells but was additionally able to kill neighboring untransfected cells. Therefore, the bystander effect greatly amplifies the efficacy of suicide gene therapy for cancer.

Apoptosis is an important biological phenomenon in tumor treatment. In the present cell apoptosis experiment, 5-FC and/or GCV treatment significantly decreased cell viability in HUVEC and BEL-7402 cells, but not in HepG2 cells. Treatment with 5-FC combined with GCV induced a more marked decrease in cell viability compared with either prodrug alone.

In order to observe the antitumor effect in vivo, $\mathrm{HCC}$ nude mouse models were established using human BEL-7402 cells. The results of the present study revealed that compared with the untransfected groups, tumors consisting of BEL-7402 cells expressing the $C D g l y T K$ gene were significantly suppressed by GCV and/or 5-FC treatment in vivo.

Angiogenesis is closely correlated with tumor growth, invasion and metastasis (38). MVD protein, which is released by tumor and stroma cells, has a significant role in angiogenesis (39). A number of proteins, including cluster of differentiation 31, cluster of differentiation 34, Factor VIII and cluster of differentiation 105, are markers of angiogenesis (40). As cluster of differentiation 34 is more sensitive and specific compared with other markers used for staining endothelial cells induced by tumor neovascularization (41), the present study assessed MVD by the presence of cluster of differentiation 34. The results of the present study revealed that the MVD of tumors was decreased by treatment with prodrugs (GCV, 5-FC or GCV+5FC) in the Ad-VEGFp-CDglyTK-transfected group, but not in the untransfected group. This result confirmed that the double suicide genes regulated by VEGFp are able to suppress tumor growth and angiogenesis in vivo.

In conclusion, the results of the present study indicate that the VEGFp-mediated double suicide gene system is able to effectively inhibit human HCC cells and vascular endothelial cells in vitro and in vivo. Expression of the CDglyTK gene under the control of the VEGFp may represent a promising gene therapy approach for the treatment of $\mathrm{HCC}$, aiming to improve long-term patient survival rates.

\section{Acknowledgements}

The present study was supported by a grant from the Natural Science Foundation of Guangdong Province (grant no., S2013010015998) and the National High Technology Research and Development Program ('863' Program) of China (grant no. 2001AA217171).

\section{References}

1. El-Serag HB and Rudolph KL: Hepatocellular carcinoma: Epidemiology and molecular carcinogenesis. Gastroenterology 132: 2557-2576, 2007.

2. Bruix J, Boix L, Sala M and Llovet JM: Focus on hepatocellular carcinoma. Cancer Cell 5: 215-219, 2004.

3. Llovet JM, Burroughs A and Bruix J: Hepatocellular carcinoma. Lancet 362: 1907-1917, 2003.

4. Wang CH, Wey KC, Mo LR, Chang KK, Lin RC and Kuo JJ: Current trends and recent advances in diagnosis, therapy, and prevention of hepatocellular carcinoma. Asian Pac J Cancer Prev 16: 3595-3604, 2015.

5. Bhayani NH,Jiang Y,Hamed O,KimchiET,Staveley-O'Carroll KF and Gusani NJ: Advances in the pharmacologic treatment of hepatocellular carcinoma. Curr Clin Pharmacol 10: 299-304, 2015.

6. Boucher PD, Im MM, Freytag SO and Shewach DS: A novel mechanism of synergistic cytotoxicity with 5-fluorocytosine and ganciclovir in double suicide gene therapy. Cancer Res 66: 3230-3237, 2006.

7. Fogar P, Greco E, Basso D, Navaglia F, Plebani M and Pedrazzoli S: Killer genes in pancreatic cancer therapy. Cell Mol Biol (Noisy-le-grand) 51: 61-76, 2005.

8. Su GQ, Su G and Huang ZH: Adenovirus-mediated tissue-targeted expression of the CDglyTk gene for the treatment of breast cancer. Mol Med Rep 6: 321-329, 2012.

9. Xu F, Li S, Li XL, Guo Y, Zou BY, Xu R, Liao H, Zhao HY Zhang Y, Guan ZZ and Zhang L: Phase I and biodistribution study of recombinant adenovirus vector-mediated herpes simplex virus thymidine kinase gene and ganciclovir administration in patients with head and neck cancer and other malignant tumors. Cancer Gene Ther 16: 723-730, 2009.

10. Rainov NG: A phase III clinical evaluation of herpes simplex virus type 1 thymidine kinase and ganciclovir gene therapy as an adjuvant to surgical resection and radiation in adults with previously untreated glioblastoma multiforme. Hum Gene Ther 11, 2389-2401, 2000.

11. Kong H, Tao L, Qi K, Wang Y, Li Q, Du J and Huang Z: Thymidine kinase/ganciclovir and cytosine deaminase/5-fluorocytosine suicide gene therapy-induced cell apoptosis in breast cancer cells. Oncol Rep 30: 1209-1214, 2013.

12. Qu L, Wang Y, Gong L, Zhu J, Gong R and Si J: Suicide gene therapy for hepatocellular carcinoma cells by survivin promoter-driven expression of the herpes simplex virus thymidine kinase gene. Oncol Rep 29: 1435-1440, 2013.

13. Sia D, Alsinet $C$, Newell $P$ and Villanueva A: VEGF signaling in cancer treatment. Curr Pharm Des 20: 2834-2842, 2014. 
14. Bertolini F, Shaked Y, Mancuso P and Kerbel RS: The multifaceted circulating endothelial cell in cancer: Towards marker and target identification. Nat Rev Cancer 6: 835-845, 2006.

15. Suh YG, Lee EJ, Cha H, Yang SH and Seong J: Prognostic values of vascular endothelial growth factor and matrix metalloproteinase-2 in hepatocellular carcinoma after radiotherapy. Dig Dis 32: 725-732, 2014

16. Bupathi M, Kaseb A and Janku F: Angiopoietin 2 as a therapeutic target in hepatocellular carcinoma treatment: Current perspectives. Onco Targets Ther 7: 1927-1932, 2014.

17. Detwiller KY, Fernando NT, Segal NH, Ryeom SW, D'Amore PA and Yoon SS: Analysis of hypoxia-related gene expression in sarcomas and effect of hypoxia on RNA interference of vascular endothelial cell growth factor A. Cancer Res 65: 5881-5889, 2005

18. Siemann DW and Shi W: Efficacy of combined antiangiogenic and vascular disrupting agents in treatment of solid tumors. Int J Radiat Oncol Biol Phys 60: 1233-1240, 2004.

19. Huber BE, Austin EA, Good SS, Knick VC, Tibbels S and Richards CA: In vivo antitumor activity of 5-fluorocytosine on human colorectal carcinoma cells genetically modified to express cytosine deaminase. Cancer Res 53: 4619-4626, 1993.

20. Weidner N, Folkman J, Pozza F, Bevilacqua P, Allred EN, Moore DH, Meli S and Gasparini G: Tumor angiogenesis: A new significant and independent prognostic indicator in early-stage breast carcinoma. J Natl Cancer Inst 84: 1875-1887, 1992.

21. Scripture CD and Figg WD: Drug interactions in cancer therapy. Nat Rev Cancer 6: 546-558, 2006.

22. Zarogoulidis P, Darwiche K and Sakkas A, Yarmus L, Huang H, Li Q, Freitag L,Zarogoulidis K and Malecki M: Suicide gene therapy for cancer - current strategies. J Genet Syndr Gene Ther 4: 2013.

23. Rogulski KR, Wing MS, Paielli DL, Gilbert JD, Kim JH and Freytag SO: Double suicide gene therapy augments the antitumor activity of a replication-competent lytic adenovirus through enhanced cytotoxicity and radiosensitization. Hum Gene Ther 11: 67-76, 2000.

24. Fischer U, Steffens S, Frank S, Rainov NG, Schulze-Osthoff K and Kramm CM: Mechanisms of thymidine kinase/ganciclovir and cytosine deaminase/5-fluorocytosine suicide gene therapy-induced cell death in glioma cells. Oncogene 24: 1231-1243, 2005.

25. Qiu Y, Peng GL, Liu QC, Li FL, Zou XS and He JX: Selective killing of lung cancer cells using carcinoembryonic antigen promoter and double suicide genes, thymidine kinase and cytosine deaminase (pCEA-TK/CD). Cancer Lett 316: 31-38, 2012.

26. Fillat C, Carrió M, Cascante A and Sangro B: Suicide gene therapy mediated by the Herpes Simplex virus thymidine kinase gene/Ganciclovir system: Fifteen years of application. Curr Gene Ther 3: 13-26, 2003.

27. Kang NH, Hwang KA, Yi BR, Lee HJ, Jeung EB, Kim SU and Choi KC: Human amniotic fluid-derived stem cells expressing cytosine deaminase and thymidine kinase inhibits the growth of breast cancer cells in cellular and xenograft mouse models. Cancer Gene Ther 19: 412-419, 2012.

28. Konopka K, Spain C, Yen A, Overlid N, Gebremedhin S and Düzgünes N: Correlation between the levels of survivin and survivin promoter-driven gene expression in cancer and non-cancer cells. Cell Mol Biol Lett 14: 70-89, 2009.
29. Tan HY, Wang N, Tsao SW, Zhang Z and Feng Y: Suppression of vascular endothelial growth factor via inactivation of eukaryotic elongation factor 2 by alkaloids in Coptidis rhizome in hepatocellular carcinoma. Integr Cancer Ther 13: 425-434, 2014

30. Li XM, Tang ZY, Zhou G, Lui YK and Ye SL: Significance of vascular endothelial growth factor mRNA expression in invasion and metastasis of hepatocellular carcinoma. J Exp Clin Cancer Res 17: 13-17, 1998.

31. Ranieri G, Ammendola M and Marech I, Laterza A, Abbate I, Oakley C, Vacca A, Sacco R and Gadaleta CD: Vascular endothelial growth factor and tryptase changes after chemoembolization in hepatocarcinoma patients. World J Gastroenterol 21: 6018-6025, 2015.

32. Suzuki K, Hayashi N, Miyamoto Y, Yamamoto M, Ohkawa K, Ito Y, Sasaki Y, Yamaguchi Y, Nakase H, Noda K, et al: Expression of vascular permeability factor/vascular endothelial growth factor in human hepatocellular carcinoma. Cancer Res 56: 3004-3009, 1996.

33. Zhan P, Qian Q and Yu LK: Serum VEGF level is associated with the outcome of patients with hepatocellular carcinoma: A meta-analysis. Hepatobiliary Surg Nutr 2: 209-215, 2013.

34. Cipriani G and Mazzanti R: Treatment with inhibitors of angiogenesis in advanced hepatocellular carcinoma: A new tool in our hands or simply a hope? Dig Liver Dis 37: 230-231, 2005.

35. Ma J, Li M, Mei L, Zhou Q, Liu L, Yu X and Che G: Double suicide genes driven by kinase domain insert containing receptor promoter selectively kill human lung cancer cells. Genet Vaccines Ther 9: 6, 2011.

36. Zhang JH, Wan MX, Yuan JY and Pan BR: Do there exist synergistic antitumor effects by coexpression of herpes simplex virus thymidine kinase with cytokine genes on human gastric cancer cell line SCG7901? World J Gastroenterol 10: 147-151, 2004.

37. Qiang L, Yanping L, Zonghai H, Fei C, Zhou L and Jinlong Y: Study of the mechanism of bystander effect of KDR-CDglyTK system mediated by adenovirus for the treatment of gastric cancer. Cell Biochem Biophys 67: 1021-1027, 2013.

38. Zhang W, Zhao CG, Sun HY, Zheng WE and Chen H: Expression characteristics of KAI1 and vascular endothelial growth factor and their diagnostic value for hepatocellular carcinoma. Gut Liver 8: 536-542, 2014.

39. Zhai LL, Wu Y, Huang DW and Tang ZG: Increased matrix metalloproteinase-2 expression and reduced tissue factor pathway inhibitor-2 expression correlate with angiogenesis and early postoperative recurrence of pancreatic carcinoma. Am J Transl Res 7: 2412-2422, 2015.

40. Zou Y, Guo CG and Zhang MM: Inhibition of human hepatocellular carcinoma tumor angiogenesis by siRNA silencing of VEGF via hepatic artery perfusion. Eur Rev Med Pharmacol Sci 19: 4751-4761, 2015.

41. Huang J, Ma X, Chen X, Liu X, Zhang B, Minmin L, Nie W, Zhang L and Liu L: Microvessel density as a prognostic factor in bladder cancer: A systematic review of literature and meta-analysis. Cancer Biomark 14: 505-514, 2014. 